\title{
Biosensor for determining average iron content of ferritin by measuring its optical dispersion
}

\author{
Ruchi Gupta $^{*}$,, Nasser A. Alamrani ${ }^{\mathrm{b}}$, Gillian M. Greenway ${ }^{\mathrm{b}}$, Nicole Pamme ${ }^{\mathrm{b}}$, Nicholas J. Goddard ${ }^{\mathrm{c}}$ \\ ${ }^{\mathrm{a}}$ School of Chemistry, University of Birmingham, Birmingham, B15 2TT, UK, ${ }^{\mathrm{b}}$ Department of \\ Chemistry and Biochemistry, University of Hull, Hull, HU6 7RX, UK, ${ }^{\mathrm{C}}$ Process Instruments (UK) \\ Ltd, March Street, Burnley, BB12 0BT, UK
}

\begin{abstract}
Average iron content of ferritin has a potential to serve as a biomarker for early identification of high-risk trauma patients at point-of-care (PoC). Appropriate therapies can then be administered to reduce morbidity and mortality. Currently, protein and iron levels are measured separately using enzyme-linked immunosorbent assay (ELISA) and UV or atomic absorption spectroscopy (AAS) respectively, but the use of two completely different methods adds to the complexity and analysis time of the combined measurement. As a result, these methods are unsuitable for PoC analysis. To address this gap, we report a biosensor for measuring the average iron content of ferritin in a single step. The biosensor was based on a dye-doped leaky waveguide (LW), which operates in the entire visible wavelength range, and hence allowed the measurement of differences in the optical dispersion of ferritin and apoferritin to determine the average iron content of the protein. The LW biosensor comprised a 1.54 micron thick mesoporous chitosan slab waveguide with immobilized antibodies against ferritin/ apoferritin to measure the optical dispersion of $110 \mathrm{nM}$ protein. Based on the baseline noise, the limit of detection for this method is $\sim 700 \mathrm{pM}$ for ferritin/ apoferritin. The biosensor has a significant potential for PoC measurement of the average iron content of serum ferritin and, in future, the total protein cencentration.
\end{abstract}

Keywords: Waveguide, biosensor, chitosan, mesoporous, optical dispersion, ferritin, point-of-care, microfluidic.

\section{INTRODUCTION}

Ferritin is an iron storage protein that can accommodate up to 4500 iron atoms in its core ${ }^{1}$. Under normal circumstances, ferritin is primarily found in cells, with little present in serum. The typical concentration of the protein in serum is between 40 and $650 \mathrm{pM}^{2}$, and is a measure of the body's iron stores. Elevated ferritin in serum is indicative of cellular damage $^{3}$, and has a potential to serve at point-of-care (PoC) as a biomarker for early identification of high-risk trauma patients and hence administer appropriate therapies to reduce morbidity and mortality. So far, however, the correlation between the concentration of iron storage protein in serum and other markers (e.g. IL-6 and CRP) of injury has not been established. One possible reason for this may be that the concentration of the former is currently measured using enzyme-linked immunosorbent assay (ELISA), which provides the combined ferritin and apoferritin concentration because the iron content in the core of the protein does not alter its ability to bind to the corresponding antibody. This implies that it is important to measure both the total concentration of ferritin and apoferritin in serum and the relative concentration of the two proteins. The latter can be determined by measuring the average iron content of the protein. Iron and protein may be assayed separately using methods such as UV or atomic absorption spectroscopy (AAS) and ELISA respectively, but the use of two completely different methods adds to the complexity and analysis time of the combined measurement, thus making these techniques unsuitable for PoC analysis.

This work reports a leaky waveguide (LW) biosensor for determining the average iron content of ferritin that does not rely on separate determination of iron and protein, but instead uses the differences in optical dispersion between ferritin and apoferritin to perform this measurement in a single step ${ }^{4}$. The imaginary refractive index of ferritin is strongly wavelength dependent because the iron present in the core of the protein has a stronger absorbance in the blue than red region of the visible spectrum. This implies that, based on the Kramer-Kronig relation, the real refractive index of ferritin is also strongly wavelength dependent. In contrast, the real refractive index of apoferritin is weakly dependent on wavelength in the visible region. Measuring the visible dispersion of ferritin/ apoferritin is therefore a measure of the average iron content of the protein. The LW comprised a 1.54 micron thick hydrogel (in this case, mesoporous chitosan) film spin-coated onto a glass substrate. At a specific angle of incidence (called the resonance angle), light is partially confined in the hydrogel film by total internal reflection (TIR) and Fresnel reflection at the hydrogel/sample and 
hydrogel/glass interfaces respectively. The resonance angle is a function of the refractive index of the waveguide and sample for a selected wavelength of light and waveguide thickness, and appears as a dip in the reflectivity curve of a LW with dye-doped waveguide. LWs can operate from $320 \mathrm{~nm}$ (limited by absorption in the BK7 substrate) and $950 \mathrm{~nm}$ (limited by cut-off). Thus, the optical dispersion of a sample can be determined by measuring resonance angle versus wavelength. Immobilized anti-ferritin antibodies in the waveguide were used to increase the local concentration of the protein in the waveguide and thus permit dispersion measurements of $110 \mathrm{nM}$ ferritin/ apoferritin. Based on the baseline noise, the limit of detection for this method is $\sim 700$ pM for ferritin/ apoferritin.

At these low concentrations, the analysis time is on the order of an hour, which is too slow for PoC use. Recently we have shown that electrokinetic preconcentration of proteins can be integrated directly above LW sensors significantly to reduce both response times and limits of detection ${ }^{5}$. We have also shown that portable and low cost $3 \mathrm{D}$ printed instrumentation can provide similar performance to large bench-top instrumentation ${ }^{6}$. The combination of dispersion measurement in LWs, electrokinetic preconcentration and 3D printed instrumentation has significant potential for PoC measurement of the average iron content of serum ferritin as well as (in future) the total protein concentration.

\section{EXPERIMENTAL}

The materials used were microscope glass slides (VWR, Leicestershire, UK), glycerol, poly(ethylene glycols) (PEGs), ethanol, $0.1 \mathrm{M}$ acetic acid, (4-(2-hydroxyethyl)-1-piperazineethanesulfonic acid) (HEPES), 25\% (v:v) glutaraldehyde (GA), reactive blue 4 (RB4), N-(3-dimethylaminopropyl)-N'-ethylcarbodiimide hydrochloride (EDC), Nhydroxysulfosuccinimide sodium salt (sulfo-NHS), ferritin (F4503), apoferritin (A3660), anti-ferritin (F6136) and bovine serum albumin (BSA, all: Sigma-Aldrich, Gillingham, UK), and chitosan ( $\mathrm{M}_{\mathrm{w}}: 100-300 \mathrm{kDa}$ and $90 \%$ deacytelated), glycerol (Mw: 92) and Decon 90 (all: Fisher, Loughborough, UK).

The glass slides were cut into 25.4 by $25.4 \mathrm{~mm}$ squares and then cleaned in Decon 90, water and ethanol for $30 \mathrm{~min}$ each in an ultrasonic bath. $1 \%(\mathrm{w}: \mathrm{v})$ chitosan was dissolved in $0.1 \mathrm{M}$ acetic acid and spin coated at $900 \mathrm{rpm}$. The spin coated films were dehydrated for 3 min following which, they were rehydrated and crosslinked by immersing in $100 \mathrm{mM}$ HEPES, pH 7.4 buffer without and with $0.016 \%$ (v: v) GA for 5 min respectively. The chitosan films were dipped in 0.1 $\mathrm{mM}$ RB4 solution in HEPES buffer for $5 \mathrm{~min}$ and then washed with the buffer to obtain dye doped waveguides.

The instrumentation set-up (Figure 1 (a)) comprised a BK7 prism, white LED, CMOS camera and optical components (lenses and grating). The flowcell was CNC machined from $3 \mathrm{~mm}$ thick black PMMA forming a recessed cavity with a 4 mm wide and $0.2 \mathrm{~mm}$ deep channel and surrounded by a groove $1 \mathrm{~mm}$ wide and $0.75 \mathrm{~mm}$ deep in which was mounted an O-ring. Antibodies were immobilised in the chitosan waveguide using EDC and sulfo-NHS protocol. All solutions were prepared in $100 \mathrm{mM}$ HEPES, pH 7.4 buffer and pumped through the flow cell using a peristaltic pump (Minipuls ${ }^{\circledR} 3$, Gilson, Bedfordshire, UK) at a flow rate of $0.2 \mathrm{ml} / \mathrm{min}$.
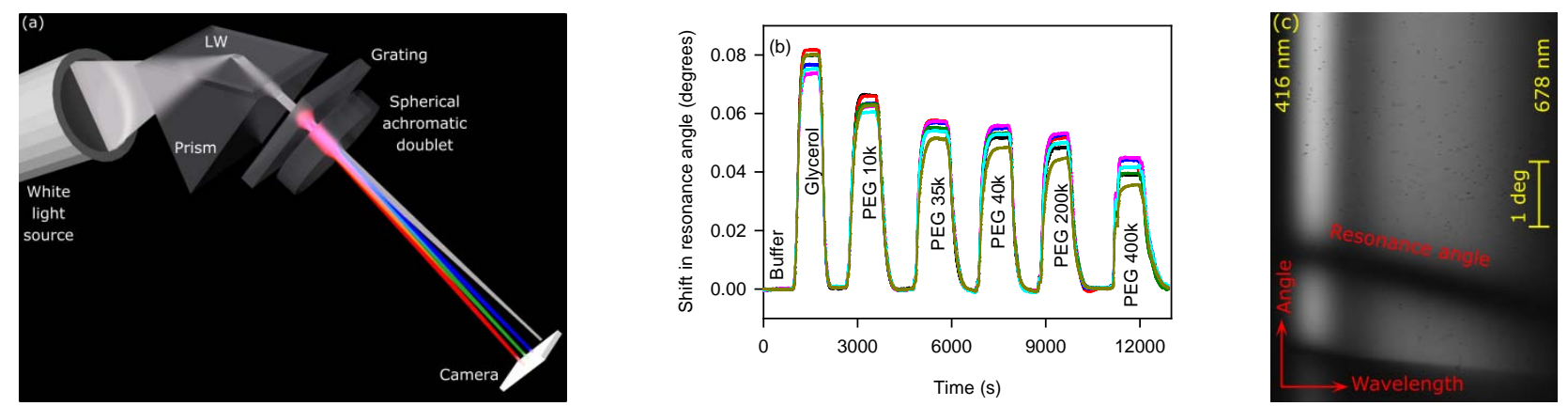

Figure 1. (a) Schematic of the instrumentation set-up (where the flow cell on top of the LW is not shown for clarity), (b) response of chitosan waveguide dried for 3 min between spin coating and subsequent rehydration to glycerol and PEGs, and (c) typical output of RB4-doped chitosan LW interrogated with white light.

\section{RESULTS AND DISCUSSION}

\subsection{Preliminary studies}

The concentration of chitosan solution was varied to identify the lowest concentration and the corresponding spin speed (1\% (w: v) polymer solution spun at $900 \mathrm{rpm}$ ) that resulted in uniform thin films capable of supporting a single optical 
LW mode. LWs comprising mesoporous hydrogel waveguide are ideal because (1) there is a complete overlap between the optical mode confined in the waveguide and biochemistry of interest, and (2) large number of antibodies can be immobilized in the hydrogel volume. The films that were allowed to dry completely after spin coating were non-porous to macromolecules where the porosity was assessed by studying the diffusion of PEGs of different molecular weights (from 10 to 400k) and a low molecular weight species, glycerol. The change in the resonance angle of the waveguide for PEGs was 84\% lower than glycerol (results not shown), suggesting that PEGs only interacted with the evanescent field of the optical mode in the waveguide. This implied that PEGs were unable to diffuse in the waveguide.

It was observed that chitosan films spin coated on glass slides were wet when removed from the chuck. The drying time between spin coating and subsequent rehydration was varied to prevent the pores in the chitosan film from collapsing during drying. A drying time of 3 min between spin coating and subsequent rehydration provided optimum compromise between well-defined dip in reflectivity at resonance angle and porosity. As shown in Figure 1 (b), the shift in the resonance angle of the chitosan waveguide dried for $3 \mathrm{~min}$ for PEGs is at least half that of glycerol. Additionally, the change in resonance angle of the fully-dried chitosan waveguide to PEG 400k was $\sim 9$ times lower than that of the film dried for 3 min after spin coating and subsequent rehydration. The estimated hydrodynamic diameter of ferritin is $\sim 12$ $\mathrm{nm}^{3}$, which is comparable to PEG 400k. Thus, the LW biosensor with the chitosan film dried for 3 min before rehydration was used to study apoferritin and ferritin, because the sensitivity of the partially-dried film was expected to be nearly an order of magnitude higher than that of the fully dried film.

\subsection{Measurement of average iron content of ferritin/ apoferritin}

Figure 1 (c) shows a typical output of a chitosan LW doped with RB4 where the $\mathrm{x}$ - and $\mathrm{y}$-axes represent wavelength and angle of incidence respectively, and the resonance angle is marked. At each wavelength, the intensity of reflected light at and the position of the resonance angle is indicative of the imaginary and real refractive index of the waveguide and sample. The resonance angle decreased as the wavelength increased from 416 to $678 \mathrm{~nm}$ because of structural and material dispersion of the LW. The measurements were made for wavelengths $>450 \mathrm{~nm}$ because the intensity of the light source was low below this wavelength.

The shift in the resonance angle versus wavelength was monitored in real-time as either apoferritin or ferritin solution was pumped over the LW comprised of a chitosan waveguide with immobilized antibodies against the protein. As shown in Figure 2 (a) and (b), the response to apoferritin $\left(\sim 0.38^{\circ}\right)$ was considerably lower than that to ferritin $\left(\sim 0.76-1.03^{\circ}\right)$, and is because of the presence of iron atoms in the core of ferritin, which increased the refractive index of the protein. Additionally, ferritin showed greater dispersion than apoferritin. This is because iron absorbs more strongly in blue than the red region of the visible spectrum. As a result, the imaginary and hence real refractive index of iron containing ferritin is strongly wavelength dependent.
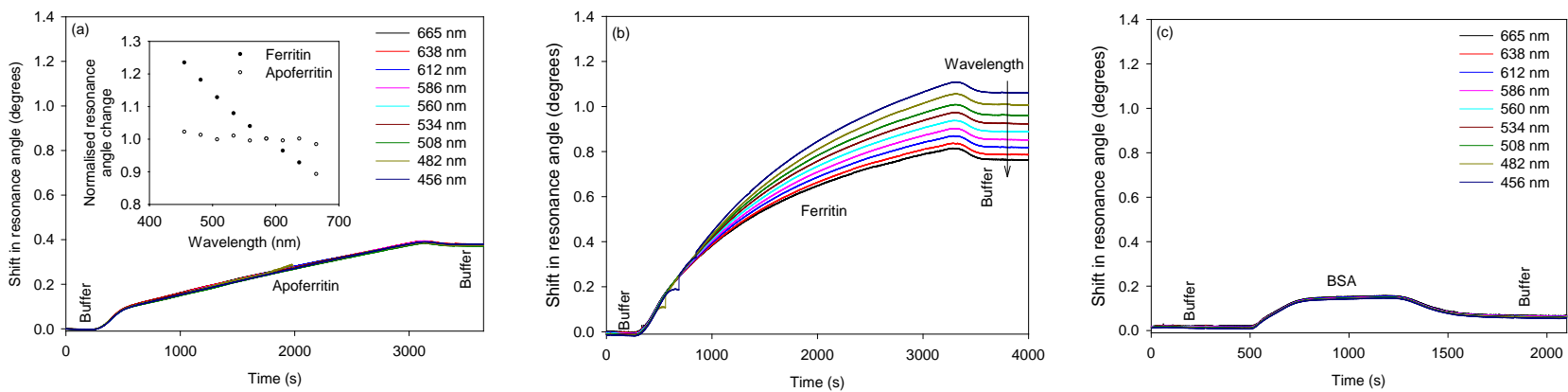

Figure 2. Sensorgrams for (a) apoferritin and (b) ferritin (where the concentration of both proteins was $110 \mathrm{nM}$ and inset shows the shift in resonance angle (normalised to the shift at $586 \mathrm{~nm}$ ) after buffer wash for both proteins as a function of wavelength), and (c) $15 \mu \mathrm{M}$ BSA.

The refractive index of the protein containing 0 and 1500 iron atoms at $587.6 \mathrm{~nm}$ has been reported to be $\sim 1.6 \times 10^{-4} \mathrm{~g}^{-1}$ and $\sim 2.55 \times 10^{-4} \mathrm{~g}^{-1}$ respectively ${ }^{7}$. The ratio of resonance angle of ferritin/ apoferritin at $587.6 \mathrm{~nm}$ was 2.29 , and assuming linear dependence between refractive index and number of iron atoms in the protein, the ferritin used in this work was estimated to contain $3375 \pm 278$ iron atoms per protein. UV-Vis spectroscopy was used independently to estimate the number of iron atoms, giving a result of 3560 per protein, which was $\sim 5.5 \%$ higher than obtained by LW.

The shift in the resonance angle at different wavelengths were normalized with respect to the shift in resonance angle at $586 \mathrm{~nm}$. A plot of normalized resonance angle shift versus wavelength for apoferritin and ferritin containing 3375 iron 
atoms is shown in the inset in Figure 2 (a). The slope of the normalized resonance angle shift for apoferritin and ferritin containing 3375 iron atoms was $-1.29 \times 10^{-4} \mathrm{~nm}^{-1}$ and $-1.62 \times 10^{-3} \mathrm{~nm}^{-1}$ respectively. Thus, the slope $\mathrm{m}$ as a function of number of iron atoms per ferritin $\left(\mathrm{N}_{\mathrm{Fe}}\right)$ is given by:

$$
\begin{aligned}
m & =-1.491 \times 10^{-3} \frac{N_{F e}}{3375}-1.29 \times 10^{-4} \\
& =-4.42 \times 10^{-7} N_{F e}-1.29 \times 10^{-4} \mathrm{~nm}^{-1}
\end{aligned}
$$

To calculate the average number of iron atoms per ferritin $\left(\mathrm{N}_{\mathrm{Fe}}\right)$, equation (1) was inverted to obtain:

$$
N_{F e}=\frac{m+1.29 \times 10^{-4}}{-4.22 \times 10^{-7}}
$$

Using the normalized resonance angle shift permitted the average number of iron atoms per ferritin ( $\left.\mathrm{N}_{\mathrm{Fe}}\right)$ to be determined independently of the ferritin concentration, while the absolute resonance angle shift was a measure of total protein concentration.

To demonstrate the selectivity of the method, BSA was introduced on the top of the chitosan waveguide with immobilised antibodies against ferritin/ apoferritin. As shown in Figure 2 (c), the shift in the resonance angle of the LW as a result of $\sim 15 \mu \mathrm{M}$ BSA was $\sim 0.14^{\circ}$ and was reduced to $0.07^{\circ}$ after a buffer wash of duration of $\sim 500 \mathrm{~s}$. In comparison, the shift in the resonance angle because of apoferritin and ferritin (both: $110 \mathrm{nM}$ ) following a similar $500 \mathrm{~s}$ buffer wash was $\sim 0.38^{\circ}$ and $\sim 0.76-1.03^{\circ}$ respectively. The measurement of ferritin/ apoferritin was taken after a buffer wash, which would largely remove any non-specifically bound sample components. This implies that the response of the chitosan waveguide with immobilised antibodies to non-specific interactions was minimal and the method is selective towards ferritin/ apoferritin.

\section{CONCLUSIONS}

We have demonstrated a leaky waveguide biosensor for ferritin/ apoferritin that permits the average iron content of the protein to be determined in a single measurement of the optical dispersion of the bound protein. The use of anti-ferritin antibodies immobilised in the waveguide provides both sensitivity and selectivity, allowing the optical dispersion of the protein to be measured at low concentrations. It was also shown that interfering species such as BSA only bound weakly to the waveguide and could be removed by washing. Current methods for determining the average iron content of ferritin require two separate measurement methods, typically ELISA for total protein and UV-Vis or atomic absorption spectroscopy for iron. This new method is a significant advance because a single measurement can provide both the average iron content and total protein concentration. We have also shown that control of the drying time of chitosan leaky waveguides before rehydration preserves the porosity of the hydrogel and provides a factor of 9 increase in sensitivity to high molecular weight species. This biosensor, when combined with integrated electrokinetic sample preparation and low cost instrumentation, is well-suited to point-of-care applications.

\section{REFERENCES}

[1] Crichton, R.R., Eason, R., Barclay, A., Bryce, C.F.A., "The subunit structure of horse spleen apoferritin: the molecular weight of the oligomer and its stability to dissociation by dilution” Biochemical Journal, 131 (4), 855 (1973).

[2] Walters, G.O., Miller, F.M., Worwood, M., "Serum ferritin concentration and iron stores in normal subjects", Journal of Clinical Pathology, 26 (10), 770 (1973).

[3] Kell, D.B., Pretorius, E., "Serum ferritin is an important inflammatory disease marker, as it is mainly a leakage product from damaged cells", Metallomics, 6 (4), 748 (2014.)

[4] Gupta, R., Alamrani, N.A., Greenway, G.M., Pamme, N., Goddard, N.J., "Method for determining average iron content of ferritin by measuring its optical dispersion", Analytical Chemistry, 91 (11), 7366 (2019).

[5] Goddard, N.J., Gupta, R., "Speed and sensitivity - integration of electrokinetic preconcentration with a leaky waveguide biosensor", Sensors and Actuators B, 301 (12), 127063 (2019).

[6] Gupta, R., Goddard, N.J., Dixon, H., Toole, N., "3D Printed Instrumentation for Point-of-Use Leaky Waveguide (LW) Biochemical Sensor", IEEE Transactions on Instrumentation and Measurement, In press, (2020).

[7] Pankowska, M., Dobek, A., "A Linear and nonlinear magneto-optics of ferritin", Journal of Chemical Physics, 131 (1), 015105 (2009)

*r.gupta.3@bham.ac.uk; phone 44121 414-6119; rguptagrouplab.com 